\title{
An Origami Microfluidic Battery: A Low-cost Activity
}

\section{Dr. Linda Vanasupa, California Polytechnic State University, San Luis Obispo}

Linda Vanasupa has been a professor of materials engineering at the California Polytechnic State University since 1991. She also serves as co-director of the Center for Sustainability in Engineering at Cal Poly. Her life's work is focused on creating ways of learning, living and being that are alternatives to the industrial era solutions-alternatives that nourish ourselves, one another and the places in which we live. Her Ph.D. and M.S. degrees are in materials science and engineering from Stanford University and her B.S. degree in metallurgical engineering from the Michigan Technological University.

Mr. Rishi Kripalani 


\title{
An Origami Microfluidic Battery: A low-cost, hands-on activity on the materials science of batteries
}

\begin{abstract}
Paper microfluidic technologies are emerging as a promising disruptive technology for low-cost sensing and detection. Researchers have developed a number of sensing and actuating devices that allow the design and creation of microfluidic devices using standard office software and equipment. These devices can be easily designed and produced in a first- or second-year engineering laboratory. This paper will discuss a novel design of a folded, paper microfluidic battery based on the work of $\mathrm{N}$. Thom et al. that can power a surface-mounted light-emitting diode. This origami design, named for the Japanese art of folding paper called origami, allows one to print and assemble postage-stamp sized paper batteries for an initial equipment investment of under $\$ 1000$ (a wax printer and micropipettes). Although the start-up cost of supplies is a few hundred dollars, the approximate cost per postage-stamp sized battery is on the order of $\$ 0.10$. The design presented here has a folded footprint of $1 \mathrm{~cm}^{2}$ and outputs an open circuit voltage of $2.5 \mathrm{~V}$ for over 15 minutes. Once printed, the dosing of electrolytes and the salt bridge, assembly and testing can be done in about 2 hours. Like standard batteries, the voltage output reflects the chemical potential difference of the electrode metals and the flow of current happens through ion transport in the electrolytes and salt bridge. This origami paper microfluidic battery is a low-cost activity that deepens the understanding of capillary action, chemical potential, and charge transport in batteries. It also represents a hands-on way to introduce students to the emerging technology of paper microfluidics.
\end{abstract}

Introduction to Paper Microfluidic Technology

Paper-based analytical devices are an emerging, ultra-low cost, open-source, scalable, portable, solution for biological and chemical sensing assays pioneered by Carrilho, Martinez, \& Whitesides (2009). These devices are made by printing hydrophobic channels on paper using the Xerox ColorQube 8580N solid-wax ink printer onto chromatography paper. Upon heating, the wax penetrates the entire thickness of the chromatography paper via capillary action. The hydrophilic channel is therefore defined by the wax that has infused the paper from the top surface, where it is originally printed, to the bottom side.

An example of a figure of a husky dog printed in wax is shown in Figure 1a. The wax is heated so that it wicks through the paper thickness (Figure 1b) to form a hydrophobic barrier that constrains the liquid analyte (Figure 1c). The fibered, cellulose chromatography paper substrates functions as a "pump" that pulls small volumes, typically microliters $(\mu \mathrm{L})$, of aqueous analyte through the hydrophilic (non-wax) channels through capillary action (Figure 1d). The analyte can be thus "pumped" toward regions with pre-deposited reagents, providing a chemical sensing platform that can be customized for the analyte. The analyte can be qualitatively or quantitatively characterized through color of the reaction product or other means, such as sensing current from an electrochemical reaction (Li, Ballerini \& Shen, 2012). This technology platform has the potential to serve as an ultra-low cost sensor for disease vectors or toxins; upon completion of the test, the paper device, typically on the order of $\mathrm{cm}^{2}$ area, can be burned to eliminate hazardous waste. 


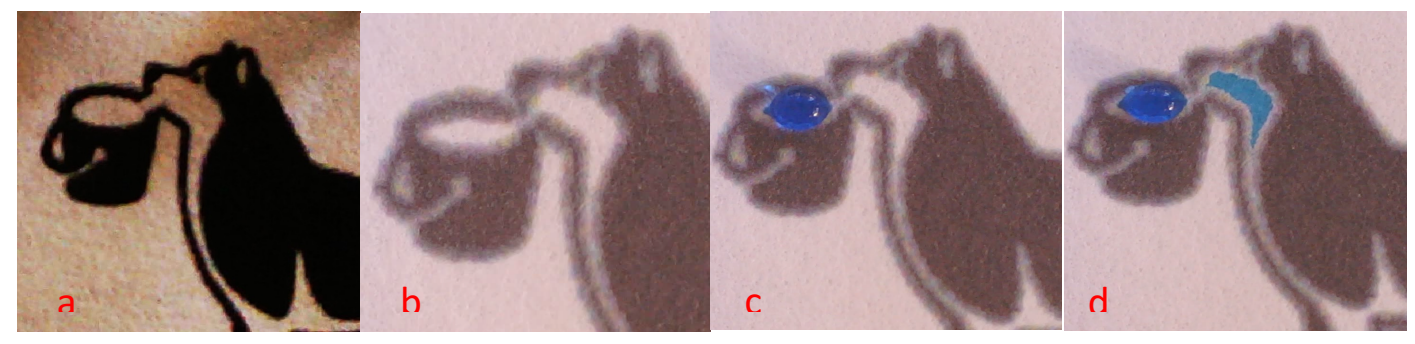

Figure 1. a. Printed wax image on chromatography paper. b. Heated image where wax has spread and wicked through the paper to form hydrophobic regions through the thickness of the paper. c. Water dyed with blue food coloring is contained in the hydrophobic regions. $d$. The water is "pumped" into the regions by capillary action of the chromatography paper fibers.

This paper describes a simple, low cost design of one such paper microfluidic device that can work in conjunction with a paper microfluidic sensor--a folded paper microfluidic battery. We developed this design, which we describe as an "origami" design. Origami is the word for the Japanese art of paper folding; since the bulk of the battery is folded paper, we call it an "origami" design. The folding circumvents the alignment challenges of the pioneering microfluidic battery design by Thom et al. $(2012,2013)$. This origami battery provides sufficient power to light a small, surface-mounted light emitting diode. This activity can be completed in under three hours from an existing origami layout file.

The learning objectives of such an activity are to:

1. Explore the connection between molecular structure of paraffin and cellulose paper and their properties of hydrophobicity and capillarity;

2. Use the difference in the chemical potential of metals in galvanic cells to produce electric power;

3. Learn how ionic conduction through electrolytes and a salt bridge can produce an electric current;

4. Discover how wax printing can be used to make a paper microfluidic device;

5. Apply the Nernst equation to the open circuit voltage of a battery.

\section{Battery Operation}

Our origami battery is based on the paper microfluidic battery design, first published by Thom et al. $(2012,2013)$. To understand the design, one must first recall how a battery works. Batteries produce a flow of charge from the natural difference in chemical potential $(\Delta G)$ of two metal electrodes. In Thom et al.' design, aluminum (Al) and silver (Ag) electrodes are used. As typical with batteries, the each metal electrode is electrically separated from the other in what is called a half-cell. Within each half-cell, the metal electrode is in contact with an aqueous solution of compatible, mobile ions called an electrolyte. Oxidation and reduction reactions take place at the different electrodes. If it were not for the salt bridge that connects the two half cells, the oxidation and reduction reactions at the electrode surfaces within the half cells would result in an imbalance of charge between the half-cells. As described below, the salt bridge allows the flow of ions from one half-cell to the other to balance out the charge imbalance; in our case, the ionic bridge consists of sodium nitrate $\left(\mathrm{NaNO}_{3}\right)$. A conceptual picture of such a battery is shown in 
Figure 2. This battery, or equivalently, "galvanic cell," is able to provide a flow of electrons when the Al and Ag electrode are electrically connected through an external load. Figure 2 shows a conceptual voltmeter (V) connected externally between the electrodes.

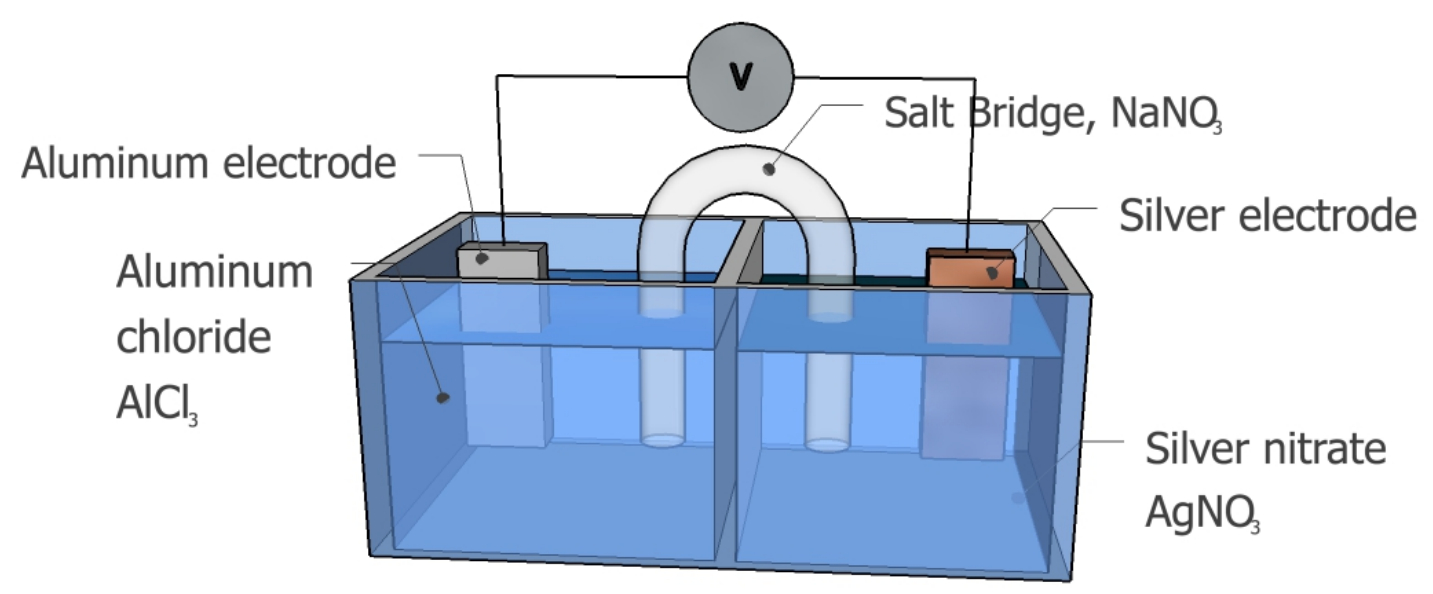

Figure 2. Concept of an Al/Ag galvanic cell. The salt bridge provides a flow of ions to offset the charge imbalance created by the oxidation and reduction reactions within the half cells (Figure 3).

Because of the difference in chemical potential of the $\mathrm{Al}$ and Ag electrode, there is an energetic driving force (i.e., electric potential) that can be translated into electrical energy through a flow of electrons. This exchange of energy occurs through neutral aluminum atoms of the Al electrode (Al) oxidizing into $\mathrm{Al}^{3+}$ ions and entering the $\mathrm{AlCl}_{3}$ electrolyte; because the ionizing of the neutral $\mathrm{Al}$ atoms provides free electrons, the aluminum electrode is therefore the anode. The negatively-charged nitrate $\left(\mathrm{NO}_{3}{ }^{-}\right)$ions in the salt bridge are drawn into the electrolyte by the positive charge created by the addition of $\mathrm{Al}^{3+}$. These nitrate ions neutralize the positive charge created by the positive $\mathrm{Al}^{3+}$ ions (Figure 3 ). Simultaneously, to offset the loss of the nitrate ions to the anode, nitrate ions enter the salt bridge from the silver nitrate electrolyte near the Ag electrode, the cathode; this also offsets the buildup of negative charge caused by $\mathrm{Ag}^{+}$from the electrolyte being reduced to Ag at the Ag surface.

The chemical reactions in the cell are:

$$
\begin{aligned}
& A l \rightarrow A l^{3+}+3 e^{-} \quad E_{o x}^{\circ}=+1.662 \text { Volts } \\
& \mathrm{Ag}^{+} \rightarrow \mathrm{Ag}+\mathrm{e}^{-} \quad E_{\text {red }}^{\circ}=+0.800 \text { Volts } \quad \text { (Vanysek, 2012) } \\
& A l+3 A^{+} \rightarrow A l^{3+}+3 A l \quad \Delta E \circ=+2.462 \text { Volts }
\end{aligned}
$$


This is normally notated in the following way:

$$
\mathrm{Al}\left|\mathrm{Al}^{3+}(\mathrm{aq}) \| \mathrm{Ag}^{+}(\mathrm{aq})\right| \mathrm{Ag}
$$

where (aq) indicates that the ion is in aqueous form.

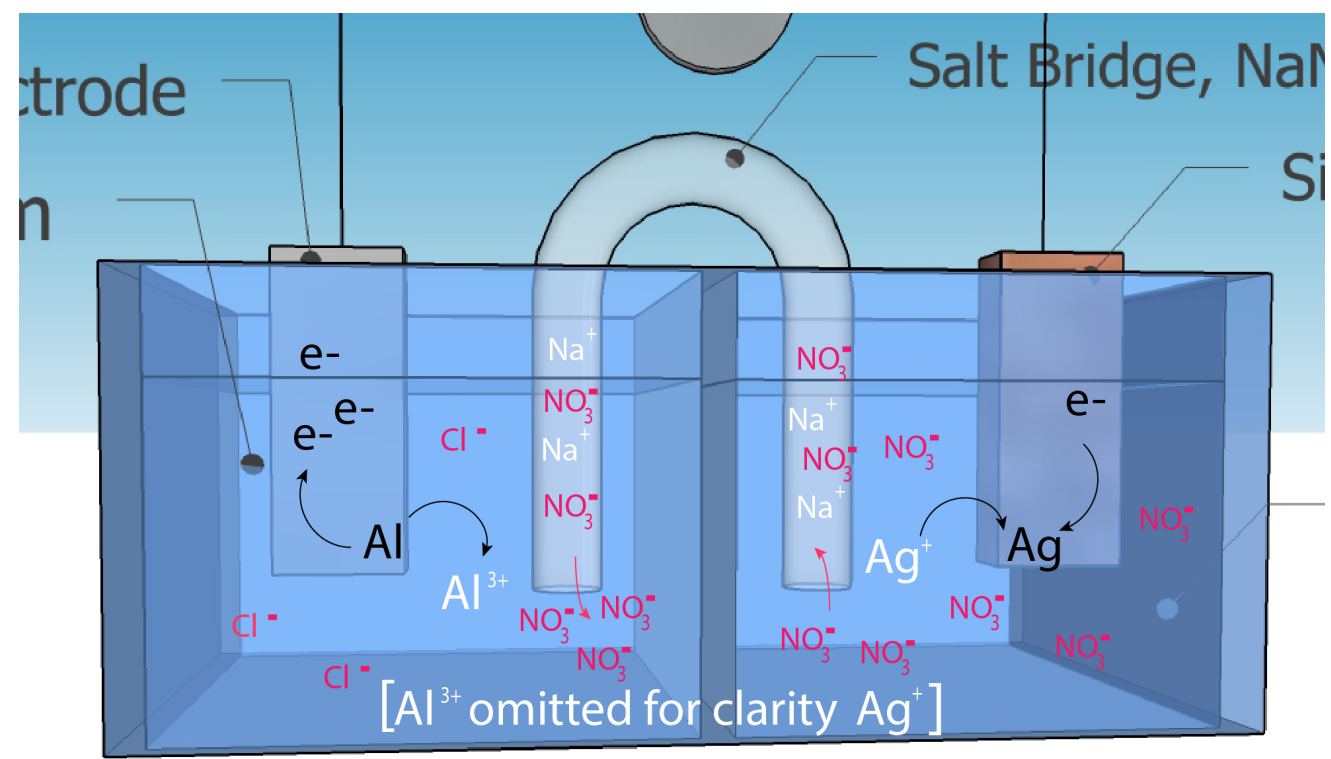

Figure 3. Illustration of anions (red, -) and cations (white, +) exchanged between the electrodes, electrolytes and salt bridge when the electrodes are externally connected. The cations in the electrolytes are omitted for clarity.

\section{Paper Microfluidic Batteries}

Microfluidic batteries are "galvanic cells incorporated directly into a multilayer paper-based microfluidic device." (Thom et al., 2013). A pattern of wax ink is first printed on the chromatography paper to delineate hydrophobic and hydrophilic regions. The hydrophilic regions serve as "reservoirs" for the liquid solutions that are then added to the layers; the hydrophobic regions are the walls of the reservoirs. Liquid electrolytes for each half-cell (AnodeAluminum, Cathode-Silver) are pre-deposited onto different layers of the device in the hydrophilic reservoirs; the water is evaporated before assembly. The salt bridge that connects the half-cells is similarly dosed and dried. Once the dehydrated layers are assembled, the addition of a solvent (in this case, deionized water), mobilizes the ions, and the reduction and oxidation reactions at the electrodes can proceed. Thus, these devices are "activated" or "turned on" via the addition of a fluid which facilitates the diffusion of ions in the electrolyte half-cells and the salt bridge. Determining factors of the power generated are volumetric density of ions, feature size, arrangement of cells, and choice of electrode metals.

In this design (Thom et al., 2013; Thom et al., 2012), alternating layers of hydrophobic dualsided adhesive layers and hydrophilic paper layers are assembled. The pre-patterned, waxprinted hydrophobic channels direct the added deionized water to the $\mathrm{NaNO}_{3}$ salt bridge that connects the two half-cells as pictured in the exploded sketch of Figure 4. Thom et al. add a 
small piece of Technicloth (C) directly to the metal electrode. Each piece is dosed with the appropriate electrolyte for the electrode on which it sits.

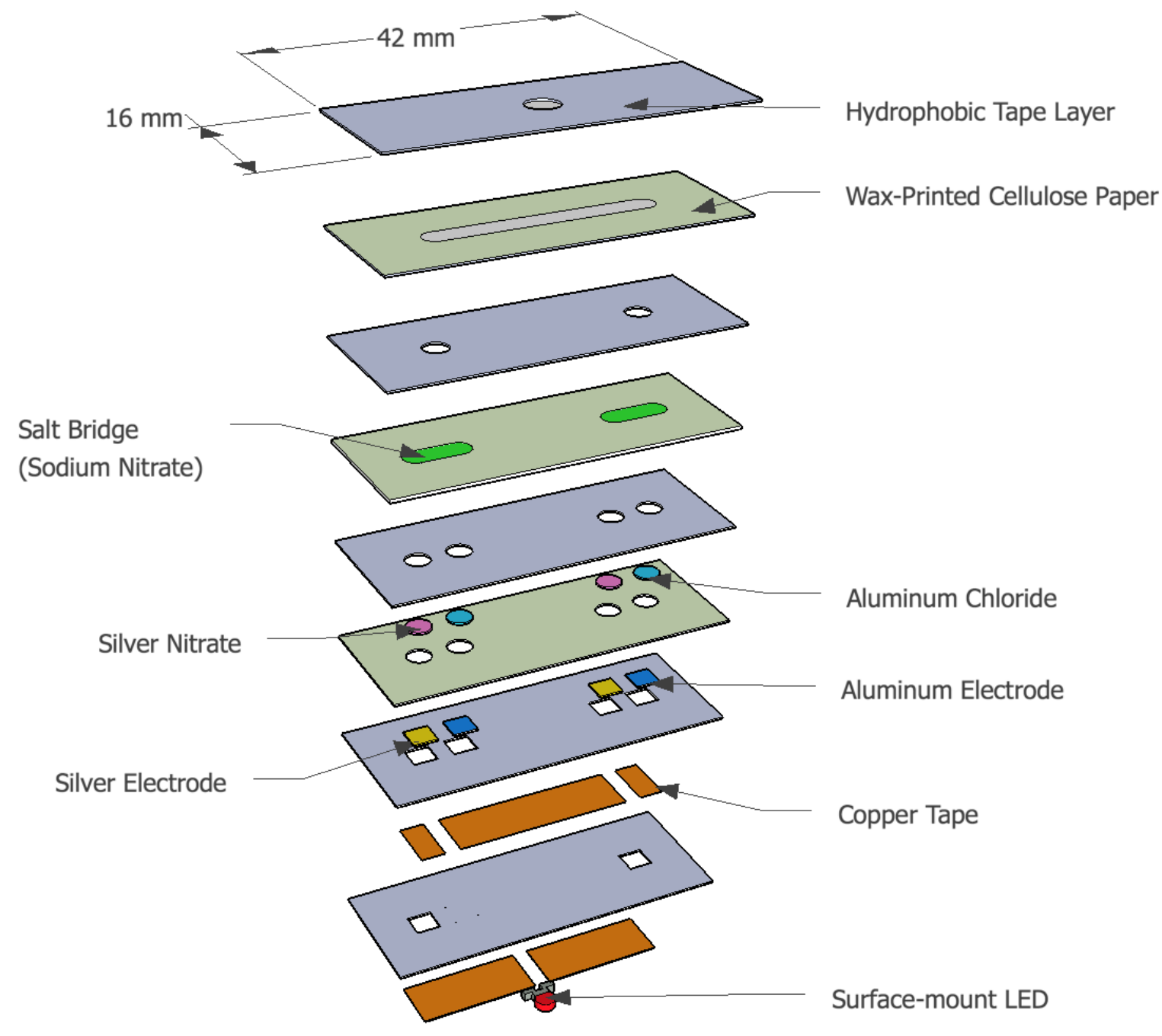

Figure 4. a Exploded sketch of two galvanic cells in series with some dimensions of the microfluidic battery. Additional dimensions of the individual layers can be found in Appendix II.

This design requires the chemical layers as indicated in Table I, listed from the top (Layer 1) to the bottom (Layer 8). These two galvanic cells in series can power a low-power, surfacemounted light emitting diode. In Table I, layers that have been patterned by mechanical stamping means that a pattern has been cut in the tape with a small circular mechanical punch.

Table I. Layer composition.

\begin{tabular}{l|l|l|l} 
Layer & Substrate & Patterning Method & Function \\
\hline
\end{tabular}




\begin{tabular}{c|l|l|l}
1 & Adhesive tape & Mechanical stamping & Sample input \\
\hline 2 & $\begin{array}{l}\text { Cellulose } \\
\text { paper }\end{array}$ & Solid-wax inkjet & Distribute sample \\
\hline 3 & Adhesive tape & Mechanical Stamping & Salt bridge input \\
\hline 4 & $\begin{array}{l}\text { Cellulose } \\
\text { paper }\end{array}$ & Solid-wax inkjet; saturated $\mathrm{NaNO}_{3}$ pre-deposited & Reconstitute salt bridge \\
\hline 5 & Adhesive tape & Mechanical stamping & Isolate electrode pads \\
\hline 6 & $\begin{array}{l}\text { Cellulose } \\
\text { paper }\end{array}$ & $\begin{array}{l}\text { Solid-wax inkjet; 3M } \mathrm{AgNO}_{3} \& 1 \mathrm{M} \mathrm{AlCl}_{3} \text { pre- } \\
\text { deposited }\end{array}$ & $\begin{array}{l}\text { Electrolyte conduction; redox } \\
\text { interface }\end{array}$ \\
\hline 7 & Adhesive tape & Mechanical stamping & Affix electrodes \\
\hline 8 & Copper tape & Mechanical cutting & Electrode circuitry
\end{tabular}

\section{Our Origami Paper Microfluidic Battery Design}

One of the challenges of the original paper microfluidic battery by Thom et al., described above and pictured in Figure 4, is the alignment between layers. We redesigned the battery to simplify the assembly. The advantage of an origami design, Figure 5, is that it largely eliminates the difficulty of aligning one layer to the next. Instead, the layers are folded onto one another like an accordion as shown in Figures 7 and 8. Because the wax penetrates the entire thickness of the chromatography paper upon heating, the top wax-printed side is roughly equivalent to the bottom, unprinted side; this makes the accordion fold (Figure 8) as effective as a layer-by-layer assembly (Figure 4). The folded battery assembly requires some way of holding it together. For quick testing, we have sandwiched the battery between plexiglass $\mathbb{C}$ using ordinary paper binder clips (Figure 7, right and Appendix), but other methods can be used.

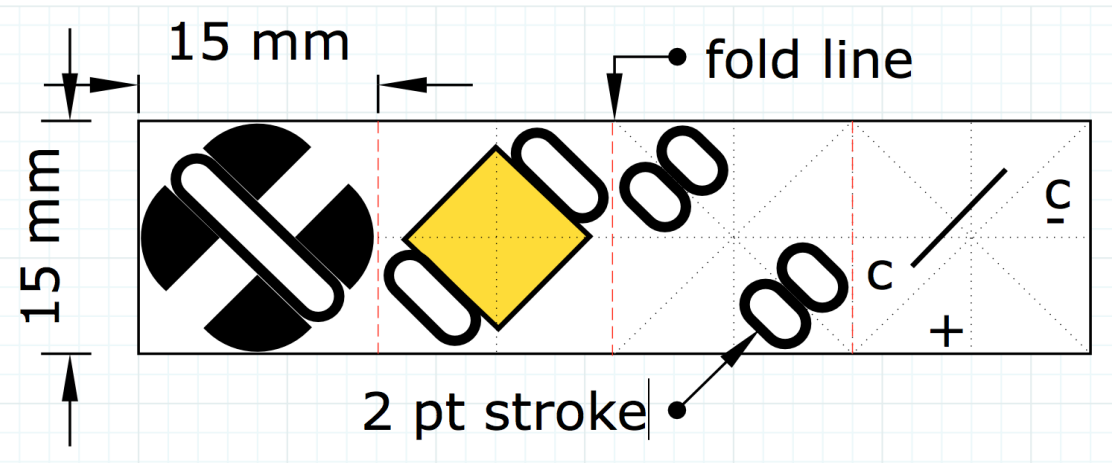

Figure 5. Print layout of an origami paper microfluidic battery with two galvanic cells in series. Red dashed lines indicate the fold lines. The "+" indicates high voltage and "-" is low voltage, with "C" indicating the location for the Al electrode. 
As printed

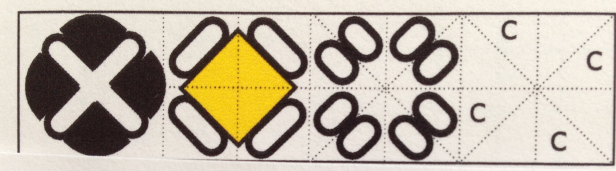

After 150C, 60C

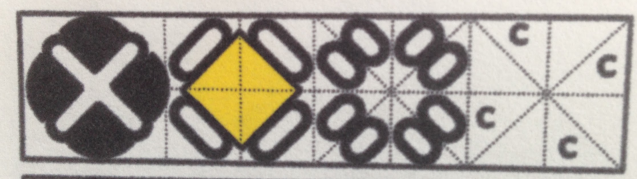

Figure 6. As printed and heated origami paper microfluidic battery design. Heating melts the paraffin wax which is drawn through the thickness of the chromatography paper and spreads through capillary action. The yellow and black solid regions are wax and thus hydrophobic; they prevent the analyte from being wicked into regions from the layer above.

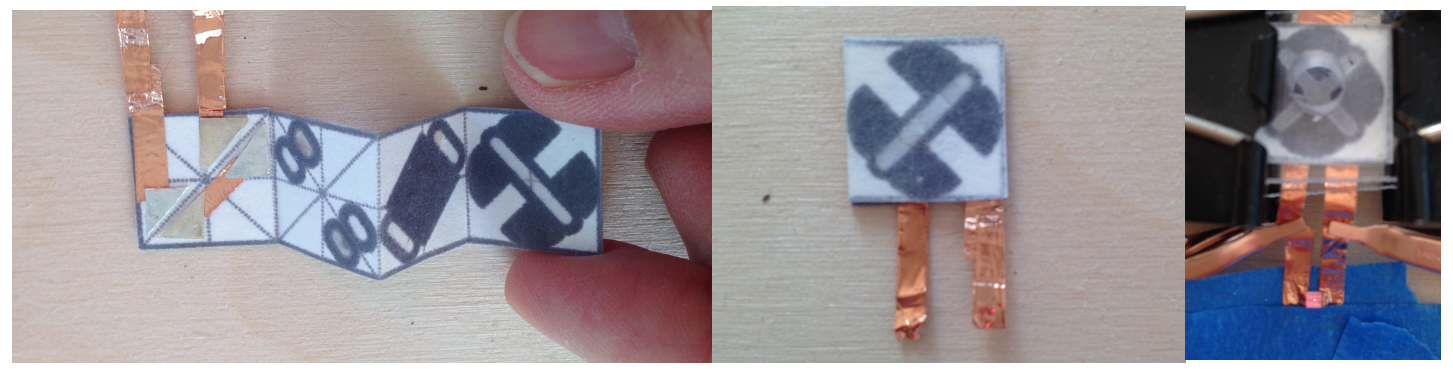

Figure 7. Battery after adding Al and Ag electrodes and dosing with electrolytes (left) and after complete folding (middle). The right image shows a red LED being lit (right, bottom-center) by an origami battery with four galvanic cells in series. The battery is sandwiched between two plexiglass plates with office-supply metal clips of $1 / 2$-inch capacity. A hole is pre-drilled in the center of the top plexiglass plate to allow the addition of water to turn on the battery.

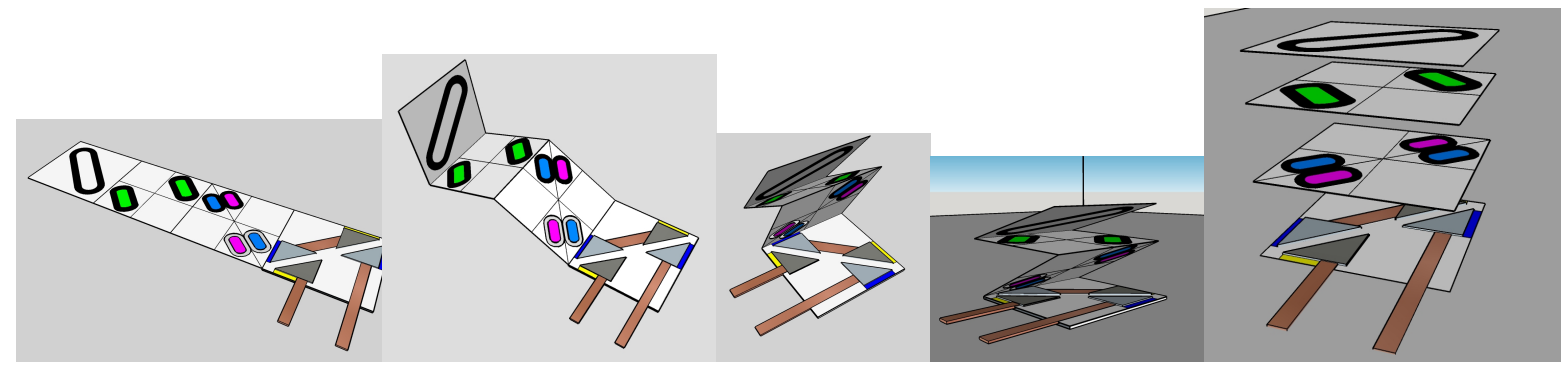

Figure 8. The folding process and the exploded view of the folded origami paper battery (right). For simplicity, this image has omitted the solid areas that block fluid from flowing where it is not wanted. The colors are used to indicate the same chemicals as indicated in Figure 4.

The advantage offered by this origami design is the simplicity of the folding; the folding process eliminates the need for cutting, aligning and taping layers together. As shown in Figure 7 (right), this design can be held together by sandwiching the battery between two pieces of plastic. The center hole in the top plastic plate is to allow the addition of the liquid.

Chemical Preparation: 
Make up $100 \mathrm{~mL}$ or less of the electrolyte solutions and the salt bridge solutions: $1 \mathrm{M} \mathrm{AlCl}_{3}, 3 \mathrm{M}$ $\mathrm{AgNO}_{3}$ and $9 \mathrm{M} \mathrm{NaNO}_{3} .100 \mathrm{~mL}$ will be a sufficient volume to fabricate at least 1000 individual batteries. (A chemical hygiene plan for making these solutions can be downloaded from the digital commons, http://digitalcommons.calpoly.edu/mate sop/.)

Battery Procedure (See Appendix I for a list of equipment and materials):

1. Layout the design to be printed or email the authors to request their design;

2. Print the layout onto Whatman\#1 chromatography paper using a Xerox ColorQube $8580 \mathrm{~N}$ wax printer;

3. Heat on a hotplate of $150^{\circ} \mathrm{C}$ for about 60 seconds or hold near a candle flame until the wax has wicked through to the backside of the chromatography paper;

4. Add $\mathrm{Al}$ and $\mathrm{Ag}$ electrodes with connecting copper tape lines;

5. Dose Technicloth $\odot$ pads and half-cell regions with $1.7 \mu \mathrm{L}$ of $1 \mathrm{M} \mathrm{AlCl}_{3}, 1.7 \mu \mathrm{L}$ of $3 \mathrm{M}$ $\mathrm{AgNO}_{3}$ and $2 \mu \mathrm{L} 9 \mathrm{M} \mathrm{NaNO}_{3}$ salt-bridge region and allow to dry;

6. Fold and compress using binder clips and Plexiglass $\subseteq$ or other stiff, transparent polymer [see Appendix I]

7. Reconstitute with $2 \mu \mathrm{L}$ deionized water or distilled water to activate.

\section{Performance of the Origami Battery}

One of the performance specifications of a battery is its open circuit voltage. This is the voltage that the battery produces when its output terminals have no electrical load (i.e., the circuit is essentially "open"). Another performance criterion is the length of time that the battery produces its open circuit voltage. As shown for this origami design in Figure 9, these two cells in series produce an open circuit voltage of about $2.6 \mathrm{~V}$ for over 13 minutes. We found that the performance parameters improved when using a small piece of Technicloth $\odot$, doped with the appropriate electrolyte and directly applied to the electrode as suggested by Thom et al. (2012).

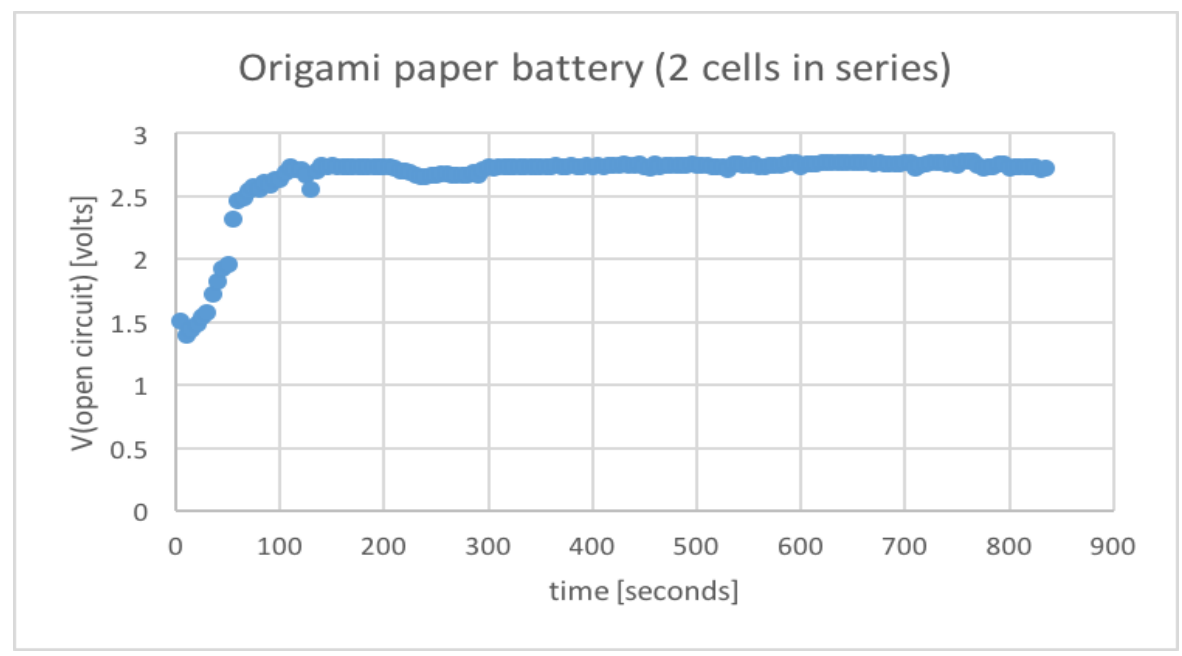

Figure 9. Performance of the origami paper microfluidic battery with two cells in series. 


\section{Discussion: Open Circuit Voltage and Battery Design}

The design choices in the layout out of the battery control its performance. For example, the amount of electrical energy theoretically available is represented by the relationship,

$$
\Delta G^{\circ}=n F E^{\circ}
$$

where $\Delta G^{\circ}$ is the change in free energy (Joules) per mole of Aluminum atoms oxidized under standard conditions ( $1 \mathrm{M}$ electrolyte, $25^{\circ} \mathrm{C}$ ), $n$ is the number of electrons exchanged per mole of Aluminum atoms oxidized, and $F$ is the Faraday constant $(96,485$ Coulombs $/ \mathrm{mol})$, and $E^{\circ}$ is the potential for the galvanic cell under standard conditions.

In our case the electrolyte concentrations differ from 1 Molar solutions; that is, they are not standard. Because we are working with very small, somewhat unknown volumes when water reconstitutes the electrolytes and salt bridge, we can consider in general how the non-standard open circuit potential, $E$, would theoretically vary with the electrolyte concentrations. This is given through the Nernst Equation,

$$
E=E^{\circ}+\frac{R T}{n F} \ln (Q)
$$

where $R$ is the gas constant $(8.314 \mathrm{~J} / \mathrm{mol} \mathrm{K}), T$ is the temperature in Kelvin, $n$ is the net number of electrons involved per mole of Aluminum oxidized, and $Q$ is a ratio of electrolyte concentrations produced by the net oxidation-reduction reaction,

For

$$
\begin{gathered}
A l+3 A g^{+} \rightarrow A l^{3+}+3 A g \\
Q=\frac{\left[A l^{3+}\right]}{\left[\operatorname{Ag}^{+}\right]^{3}}
\end{gathered}
$$

and $\left[\mathrm{Al}^{3+}\right]$ indicates the molar concentration of the $A l^{3+}$ electrolyte solution in contact with the electrode.

The origami battery design provides an opportunity for the student designers to apply these equations to change the open circuit voltage, $E$. In building these batteries, we have found that the use of an electrolyte-doped Technicloth $(\mathrm{C}$ pad on each of the electrodes increases the output current, demonstrated by the brighter intensity of the LED. We believe this increase in current output is due to reducing the internal resistance of the battery through enhanced ionic conduction provided by the electrolyte-doped Technicloth $₫$ pad in contact with the electrode.

\section{Conclusion}

This paper describes a simple design for a folded paper microfluidic battery that can be designed and built by undergraduate students using standard office equipment and simple chemistries. The activity introduces undergraduates to the emerging technology of paper microfluidic sensors and devices. It demonstrates that application of a number of concepts: the capillary effect, the Nernst relation, ionic conduction, hydrophobicity, and the energy stored in chemical potential. 
This technology holds promise as a platform for ultra low-cost sensing and detection of toxins or disease vectors; it is also a low-cost platform for undergraduates to learn about how energy is stored and discharged in batteries and the battery design parameters that influence battery performance.

\section{Acknowledgements}

The authors would like to acknowledge their gratitude for the guidance and helpful conversations with Nicole Thom and Scott Phillips. They also acknowledge the foundational exploratory work of colleagues Cory Hughes, Glenn Lee, and Jeremy Dunn.

\section{References}

Carrilho, E., Martinez, A. W., \& Whitesides, G. M. (2009). Understanding wax printing: A simple micropatterning process for paper-based microfluidics. Analytical Chemistry, 81(16), 7091-7095.

Li, X., Ballerini, D. R., \& Shen, W. (2012). A perspective on paper-based microfluidics: Current status and future trends. Biomicrofluidics, 6(1), 011301-13.

Lide, David R., ed. (2006). CRC Handbook of Chemistry and Physics (87th ed.). Boca Raton, FL: CRC Press. ISBN 0-8493-0487-3.

Thom, N. K., Yeung, K., Pillion, M. B., \& Phillips, S. T. (2012). "Fluidic batteries" as low-cost sources of power in paper-based microfluidic devices. Lab Chip, 12(10), 1768-1770.

Thom, N. K., Lewis, G. G., DiTucci, M. J., \& Phillips, S. T. (2013). Two general designs for fluidic batteries in paper-based microfluidic devices that provide predictable and tunable sources of power for on-chip assays. RSC Advances, 3(19), 6888-8.

Vanýsek, Petr (2012). "Electrochemical Series". In Haynes, William M. Handbook of Chemistry and Physics: 93rd Edition. Chemical Rubber Company. pp. 5-80. ISBN 9781439880494. 
Appendix I: List of materials, equipment, supplies.

\begin{tabular}{|c|c|c|c|}
\hline $\begin{array}{l}\text { Equipment } \\
\text { Hardware }\end{array}$ & $\begin{array}{l}\text { Amount/Q } \\
\text { uantity }\end{array}$ & Description & Cost \\
\hline Solid Ink Printer & 1 & Xerox ColorQube $8580 \mathrm{~N}$ & $\$ 599.00$ \\
\hline \multirow[t]{3}{*}{ Micropipette } & 1 & $\begin{array}{l}\text { Scilogex } 71312119 \text { MicroPette Plus Single-Channel } \\
\text { Fixed Volume Pipettor, } 10 \text { microliter Volume }\end{array}$ & $\$ 55.00$ \\
\hline & 1 & $\begin{array}{l}\text { Scilogex } 71312123 \text { MicroPette Plus Single-Channel } \\
\text { Fixed Volume Pipettor, } 100 \text { microliter Volume }\end{array}$ & $\$ 55.00$ \\
\hline & 1 & $\begin{array}{l}\text { Scilogex } 71312126 \text { MicroPette Plus Single-Channel } \\
\text { Fixed Volume Pipettor, } 500 \text { microliter Volume }\end{array}$ & $\$ 55.00$ \\
\hline \multirow[t]{3}{*}{ Micropipette Tips } & 1 & $\begin{array}{l}\text { Scilogex } 750003 \text { MicroPette Universal Pipette Tip, } 0.2- \\
10 \text { uL Volume, Clear, Sterile, } 10 \text { x } 96 \text { Rack (Pack of 960) }\end{array}$ & $\$ 69.38$ \\
\hline & & $\begin{array}{l}\text { Scilogex } 750005 \text { MicroPette Universal Pipette Tip, 10- } \\
200 \text { uL Volume, Yellow, } 10 \text { x } 96 \text { Rack (Pack of 960) }\end{array}$ & $\$ 48.01$ \\
\hline & 1 & $\begin{array}{l}\text { Scilogex } 750007 \text { MicroPette Universal Pipette Tip, 100- } \\
1000 \text { uL Volume, Clear, Bulk (Pack of 1000) }\end{array}$ & $\$ 22.08$ \\
\hline Hotplate & 1 & $\begin{array}{l}\text { Corning }{ }^{\circledR} \text { Scholar } 170, \text { AC input } 120 \mathrm{~V} \text {, US 3-pin plug, } \\
\text { plate } \times \text { L } 5 \text { in. } \times 5 \text { in. }\end{array}$ & $\$ 152.80$ \\
\hline Candle & 1 & 6" x 3" Unscented, Smokeless Cotton Wick & $\$ 7.99$ \\
\hline \multicolumn{4}{|l|}{ Software } \\
\hline SketchUp Pro & 1 & SketchUp Pro for Educators & Free \\
\hline \multicolumn{4}{|l|}{ Materials } \\
\hline Cellulose substrate & 1 & $\begin{array}{l}\text { Whatman Cellulose Chromatography Paper, Grade 1, } 100 \\
\text { sheets, } 46 \text { x } 57 \mathrm{~cm}\end{array}$ & $\$ 238.70$ \\
\hline Aluminum Chloride & $100 \mathrm{~g}$ & $\begin{array}{l}\text { anhydrous, glass bottle, ReagentPlus }{ }^{\circledR} \text {, anhydrous, } \\
\geq 99.9 \% \text { trace metals basis }\end{array}$ & $\$ 58.40$ \\
\hline Silver Nitrate & $5 \mathrm{~g}$ & Grade: ReagentPlus ${ }^{\circledR}$; Packaging: amber poly bottle & $\$ 29.40$ \\
\hline Sodium Nitrate & $5 \mathrm{~g}$ & Grade: ReagentPlus ${ }^{\circledR}$; Packaging: poly bottle & $\$ 39.80$ \\
\hline Aluminum & $1.7 \mathrm{~g}$ & $50 \times 50 \mathrm{~mm} ; 99.999 \%$ pure & $\$ 55.40$ \\
\hline Silver & $6.6 \mathrm{~g}$ & $50 \times 50 \mathrm{~mm} ; 99.9 \%$ pure & $\$ 65.70$ \\
\hline Copper Tape & 1 & $6.3 \mathrm{~mm} \mathrm{~W} \times 16.46 \mathrm{~m} \mathrm{~L}$ & $\$ 18.95$ \\
\hline TechniCloth & 1 & $\begin{array}{l}\text { TechniCloth II, TX } 1109,9 " \text { " } 9 \text { ", cellulose/polyester- } \\
\text { blend wipe, non-woven fiber with ULP treatment, } 300 \\
\text { sheets per pack, } 10 \text { packs per case } \\
\text { (1 sheet needed!) }\end{array}$ & $\$ 271.53$ \\
\hline Deionized water & 1 Liter & 38796 Sigma Aldrich & $\$ 22.69$ \\
\hline
\end{tabular}

[link to Table of materials/equipment:

https://docs.google.com/spreadsheets/d/1KjBlQOIyArf4i8Xfs-YWQIGH9L4W2FzvFilGj0Mn4M/edit?usp=sharing] 


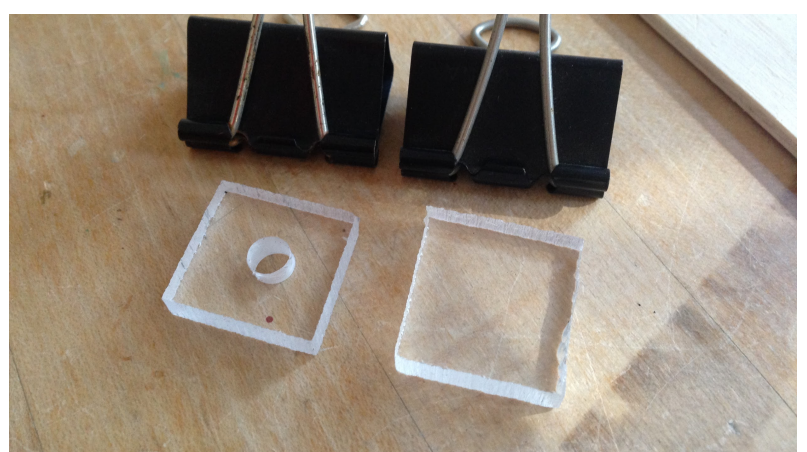

Plexiglass (C) pieces and binder clips used to quickly test the origami battery performance. These are used as pictured in Figure 7. The composition of the polymer that is used is not important. It simply needs to be stiff and transparent. Glass is a suitable alternative. 
Appendix II: Dimensions of the individual layers for the design from Figure 4.

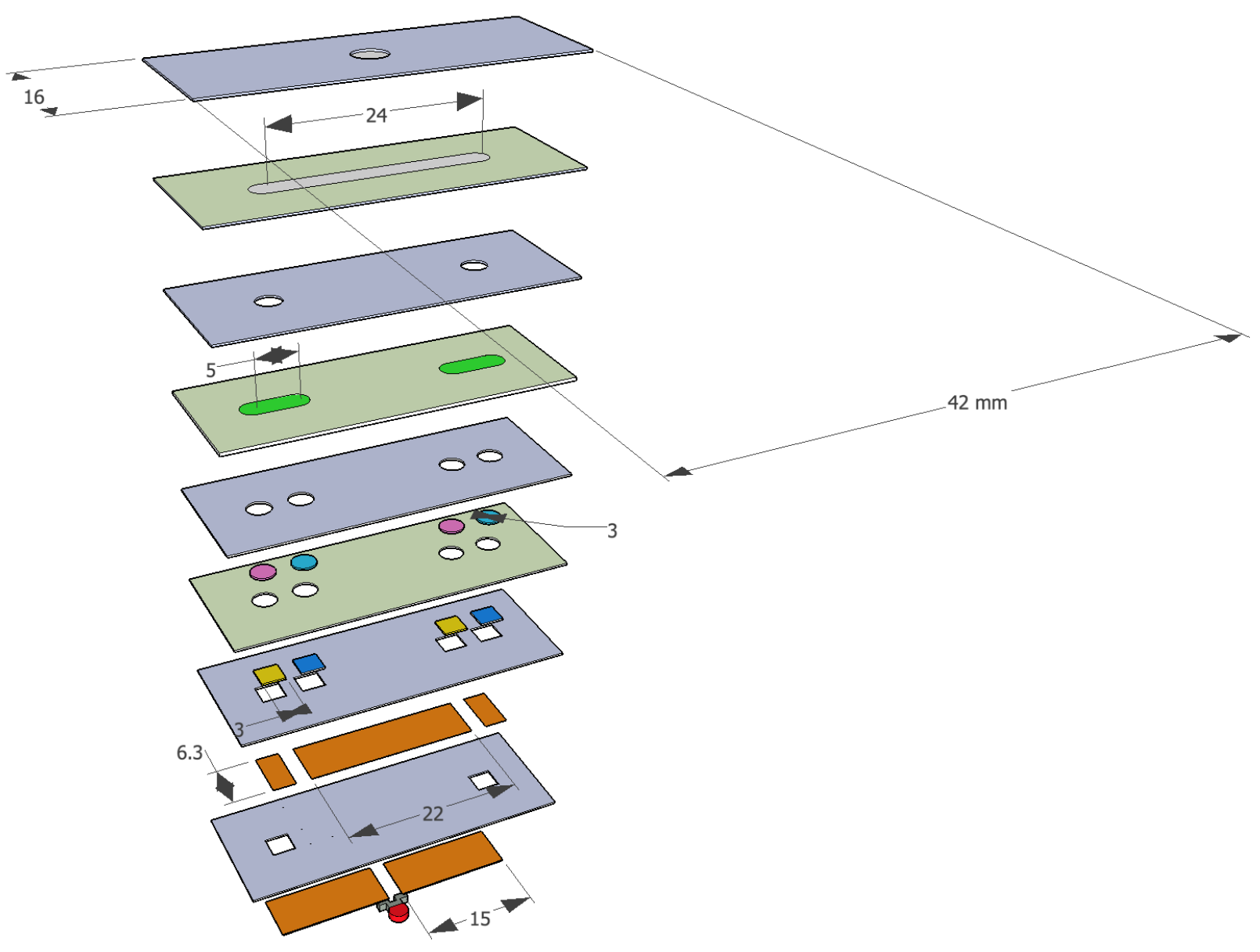

